\title{
MJN OUTCOMES OF COVID-19 DURING PREGNANCY: A SYSTEMATIC REVIEW
}

\author{
Divya Kuzhivilayil Yesodharan", Divya Raghavan ${ }^{2 *}$, Teena Jones ${ }^{3}$
}

${ }^{1}$ Department of Community and Mental Health, College of Nursing, Sultan Qaboos University, Muscat, Oman ${ }^{2}$ Department of Maternal and Child Health, College of Nursing, Sultan Qaboos University, Muscat, Oman ${ }^{3}$ Early Pregnancy Unit, East Surrey Hospital, Surrey and Sussex NHS Trust, Surrey Redhill, UK

*Corresponding Author’s Email: divyam@squ.edu.om

\begin{abstract}
Objective: This paper presents specific pregnancy outcomes of COVID-19 during pregnancy, based on the literature available until August 2020. Methods: A systematic review was conducted in PubMed, Scopus, Google Scholar, Springer, Science Direct, and the EMBASE database of publications regarding coronavirus disease (COVID-19) following the PRISMA checklist. The search terms used include: "pregnancy"; "pregnant"; "neonatal"; "neonate"; "COVID-19"; "coronavirus disease 2019" "pregnancy", "pregnant", "neonatal" and "neonate". All studies exploring the maternal and fetal outcomes among pregnant women with COVID-19 were considered for this review. Results: A total of 11 case reports on 17 pregnant women and 12 retrospective reviews of the medical records of 795 pregnant women meeting the inclusion criteria were included in the review. Of 362 deliveries, 59.3\% involved caesarean sections [CS] while $40.6 \%$ were vaginal deliveries. Of the 362 deliveries, $22.37 \%$ were preterm. Of 51 neonates, $20 \%$ were found to have low birth weight and one tested positive for SARS-CoV-2. A total of nine neonatal deaths were reported, which were not associated with the viral infection, and 15 neonates were found to be COVID-19 positive, although no indication of vertical transmission of infection was established. Conclusions: Pregnancy does not increase the risk of COVID-19 and prospective mothers should follow the same recommendations as applicable to the general population, to limit the transmission of COVID-19. Information compiled in this systematic review of COVID-19-related pregnancy outcomes may help in planning the best care in future.
\end{abstract}

Keywords: COVID-19; Pregnancy Outcome;Maternal Outcome; Foetal

\section{INTRODUCTION}

Coronavirus disease or COVID-19 is the most pressing global health issue today, affecting all sectors of society irrespective of caste, creed, ethnicity or economical differences. Although COVID-19 can affect anyone, its impact is greater on people with lower immunological status, a category that includes pregnant women (Bouaziz et al., 2020).

Pregnant women and their fetuses remain at high risk during any infectious disease breakdown. (Dashraath et al., 2020) Knowledge gained from previous SARS coronavirus (SARS-CoV) and MERS coronavirus (MERS-CoV) suggests that pregnant status increases the risk of poor outcomes (Zaigham \&Andersson, 2020). Prevention and control of COVID19 among pregnant women and the risk of its vertical transmission are growing concerns (Qiao, 2020).

\section{Aims and Objectives of the Review:}

COVID-19 is infecting many people globally and the numbers of infections and deaths per day are rapidly evolving. Yet there is limited data available on its impact upon pregnant women, and the maternal and fetal outcome (Rasmussen et al., 2020). The clinical pneumonia associated with COVID-19 were similar in pregnant women and non-pregnant adults (Rasmussen et al., 2020). The physiological changes during pregnancy, such as decreased lung capacity and other immunological adaptations, pose an increased risk of complications from respiratory illness among pregnant women (Schwartz, 2020).

Studies have shown that COVID-19 may result in fetal distress, abortion, preterm labor, and respiratory 
distress in women during pregnancy (Panahi, Amiri \& Pouy, 2020) Since a smaller number of cases are involved in the published studies, the research is inconclusive as to whether these maternal and fetal outcomes are exclusively due to COVID-19 related pneumonia or other pre-existing maternal conditions. A systematic review of the available literature as of August 2020 is therefore necessary to understand the effect of COVID-19 on pregnancy outcome among COVID-19-positive pregnant women.

\section{METHODOLOGY}

\section{Study Design}

Two reviewers conducted a comprehensive examination of relevant literature independently. The databases searched included Google Scholar, Springer, ScienceDirect, PubMed, Scopus and EMBASE. The search terms used include: "pregnancy"; "pregnant"; "neonatal"; "neonate"; "COVID-19"; "coronavirus disease 2019" "pregnancy", "pregnant", "neonatal" and "neonate". The search criteria sought studies that explore the impact of COVID-19 on maternal or foetal outcome among pregnant women, published in English and available as a free, full-text download. Unpublished reports, existing systematic reviews on similar topics and comments to the editor are excluded from this review.

The initial search revealed 500 studies and after removing 200 duplicates, a title-and-abstract screening of the remaining 300 studies was carried out. A further 200 studies were removed as the full text was either unavailable for free download or unavailable in English. After full-text screening, 77 studies were removed either because they lacked full information or did not mention specific maternal or foetal outcomes. The remaining 23 studies met the eligibility criteria and are included in this review. They comprise 11 case reports and 12 retrospective clinical data reviews. Twelve studies are from China, two from both the UK and USA and one from each of Australia, Brazil, France, Iran, Italy, and Sweden. One study does not mention its country of origin.

Data Extraction: Two reviewers independently of one another conducted the screening of the studies and care was taken to ensure that no study was counted twice. Since only a handful of studies with small sample sizes are available, the researchers did not undertake quality appraisal of the studies included. The data extracted from the studies includes study ID, research method, and place of study, sample, and mode of delivery, week of gestation at delivery, maternal outcome and foetal outcome.

Quality Analysis: Case reports and case series designs have poor methodological controls and are known to increase the risk of bias, but they continue to contribute to the body of medical knowledge. Both reviewers to ensure the quality of the included studies, used methodological quality and synthesis of case series along with case reports independently

Risk of Bias: The CARE checklist (Consensus-based Clinical Case Reporting Guideline Development), consisting of 30 items, was used to assess the quality of case reports retrieved (Gagnier et al., 2013). The STROBE checklist (Strengthening the Reporting of Observational Studies in Epidemiology), comprising 34 items, was used to assess the reliability of cohort reports (Cevallos, Egger \& Moher, 2014).

Reporting: This article followed the PRISMA checklist (Preferred Reporting Items for Systematic Review and Meta-analysis) for reporting (Moher et al., 2009).

The search strategy is displayed in Figure: 1

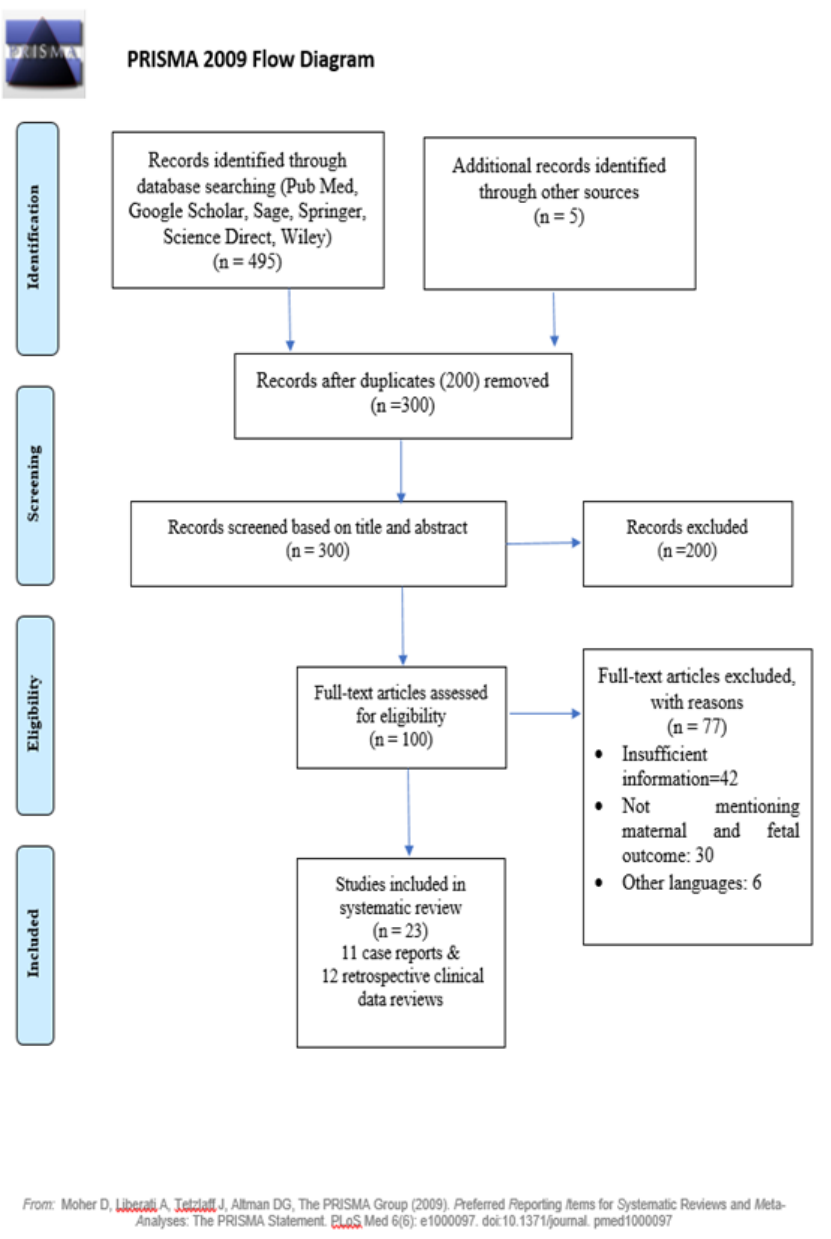

Figure: 1 PRISMA Showing the Search Strategy 


\section{RESULTS}

Though the majority of reviews were from China; studies from Australia, Brazil, France, Iran, Italy, Sweden, UK and USA make this a good database for review. We made an overview and refined review to ensure a satisfactory quality of study included in the review. The researchers tried to review all available case reports to ensure maximum domain coverage of the content reviewed. The cases described and the medical record-related articles that were retrospectively reviewed were described in sufficient detail to ensure the appropriate reporting of the content under review.

The information on following items was individually collected by the researchers from the original reports: authors' names, country, study design, number of pregnant women as sample in each study, maternal characteristics (maternal age, gestational age at admission), and mode of confirmation of COVID-19 diagnosis in terms of laboratory diagnosis and clinical diagnosis. Maternal, obstetric and neonatal outcomes were also extracted from the reviewed literature.

A total of 23 studies including a sample of 812 COVID-19 pregnant women were identified as per eligibility criteria and included in this review. The results from 11 case reports with details of 17 patients are summarized together to facilitate interpretation. These were published from January 2020 to July 2020.

Among the included studies, a laboratory-confirmed case of COVID-19 was defined as a positive result on quantitative reverse transcriptase polymerase chain reaction (RT-PCR) assay of maternal pharyngeal swab specimens. Radiological and chest CT findings to supplement diagnosis were considered to be a clinical diagnosis of COVID-19.

Maternal outcomes included information about any reported complications related to pregnancy and outcomes of pregnancy, which included mode of delivery, pregnancy loss and the number of women still pregnant at the end of the study, and other possible outcomes of pregnancy, like maternal death, IUD etc. Outcomes in Neonatal reviewed were, Apgar scores, presence of neonatal asphyxia, neonatal death, neonatal positive COVID-19 tests and other types of pneumonia requiring neonatal intensive care unit (NICU) admission and care. A worksheet was prepared to summarize the data extracted from the studies reviewed. Data entry and processing were performed with Microsoft ${ }^{\circledR}$ Office Excel 365®. Data analysis consisted of the calculation of means and percentages.

Table 1: Study Characteristics $n=812$

\begin{tabular}{|c|c|c|c|c|c|c|c|}
\hline $\begin{array}{l}\text { SI } \\
\text { No }\end{array}$ & Study ID & (n) & Research Method & Country & Lab diagnosis & Clinical diagnosis & GA at diagnosis \\
\hline 1 & $\begin{array}{l}\text { Koumoutsea EV et } \\
\text { al (2020) }\end{array}$ & 2 & Case Study Review & China & RT PCR & $\begin{array}{l}\text { Chest } \mathrm{x} \text { ray normal; } \\
\text { NO CT; Respiratory } \\
\text { parameters normal }\end{array}$ & $35+3$ WOG \\
\hline 2 & $\begin{array}{l}\text { Alzamora MC et al } \\
(2020)\end{array}$ & 1 & Case Study Review & china & RT PCR & Not done & 33 WOG \\
\hline 3 & Liu W (2020) & 3 & Case Study Review & CHina & RT PCR & Not done & $38+$ WOG \\
\hline 4 & Khan. S et al (2020) & 3 & Case Study Review & china & RT PCR & Not Done & 33 WOG;39 WOG;38+2 WOG \\
\hline 5 & $\begin{array}{l}\text { Zambrano et al } \\
(2020)\end{array}$ & 1 & Case Study Review & $\begin{array}{c}\text { Central } \\
\text { America }\end{array}$ & RT PCR & Not done & $31 \mathrm{WOG}$ \\
\hline 6 & $\begin{array}{l}\text { Kolkova Z et al } \\
(2020)\end{array}$ & 1 & Case Study Review & Sweden & RT PCR & CT scan & $32 \mathrm{WOG}$ \\
\hline 7 & $\begin{array}{l}\text { Celso T et al } \\
(2020)\end{array}$ & 2 & Case Study Review & Brazil & RT PCR & $\begin{array}{l}\text { Chest X ray; } \\
\text { CT } \operatorname{scan}(2)\end{array}$ & 32WOG; 28WOG \\
\hline 8 & Yu Y et al. & 1 & Case Study Review & China & RT PCR & Chest CT & 34 WOG \\
\hline 9 & Andrew et al (2020) & 1 & Case Study Review & Australia & RT PCR & Not done & $39+3$ WOG \\
\hline 10 & Hansen et al (2020) & 1 & Case Study Review & USA & RT PCR & Not done & $34 \mathrm{WOG}$ \\
\hline 11 & $\begin{array}{l}\text { Gonzailez et al } \\
(2020)\end{array}$ & 1 & Case Study Review & NA & RT PCR & Chest X Ray & $29+3$ WOG \\
\hline
\end{tabular}




\begin{tabular}{|c|c|c|c|c|c|c|c|}
\hline 12 & Xu L et al (2020) & 5 & $\begin{array}{l}\text { Retrospective Medical } \\
\text { records review }\end{array}$ & China & RT-PCR & $\mathrm{X}$ ray & $34+$ WOG \\
\hline 13 & Cao D et al (2020) & 10 & $\begin{array}{l}\text { Retrospective Medical } \\
\text { records review }\end{array}$ & China & RT-PCR & Pulmonary CT & $33+6$ to $40+5$ \\
\hline 14 & Zeng Y et al (2020) & 16 & $\begin{array}{l}\text { Retrospective Medical } \\
\text { records review }\end{array}$ & China & RT-PCR & Pulmonary CT & $37+5 \mathrm{WOG}$ \\
\hline 15 & $\begin{array}{l}\text { Antoun L et al } \\
(2020)\end{array}$ & 23 & $\begin{array}{l}\text { Retrospective Medical } \\
\text { records review }\end{array}$ & UK & RT-PCR & Chest Radiography & $\begin{array}{c}19=3 \mathrm{rd} \text { trimester; } 04=2 \mathrm{nd} \\
\text { trimester }\end{array}$ \\
\hline 16 & Nan Yu et al (2020) & 7 & $\begin{array}{l}\text { Retrospective Medical } \\
\text { records review }\end{array}$ & CHina & RT-PCR & $\mathrm{CT}$ on admission & $39+1 \mathrm{WOG}$ \\
\hline 17 & Nie R, et al (2020) & 33 & $\begin{array}{l}\text { Retrospective Medical } \\
\text { records review }\end{array}$ & China & RT-PCR & Not done & $\begin{array}{c}3=17-26 \mathrm{WOG} ; 28=<37 \mathrm{WOG} ; \\
2=>38 \mathrm{WOG}\end{array}$ \\
\hline 18 & $\begin{array}{l}\text { HantoushzadeH S, et } \\
\text { al (2020) }\end{array}$ & 9 & $\begin{array}{l}\text { Retrospective Medical } \\
\text { records review }\end{array}$ & Iran & RT-PCR & Not done & $\begin{array}{c}\text { Gestational age (wk) c1: } 303 / 7 \\
\text { c2: } 383 / 7 \mathrm{c} 3: 305 / 7 \mathrm{c} 4: 240 / 7 \\
\text { (undelivered) c5: } 36 \text { 0/7 c6: } 24 \\
\text { 0/7 (undelivered) c7: } 280 / 7\end{array}$ \\
\hline 19 & Liu D.et al (2020) & 15 & $\begin{array}{l}\text { Retrospective Medical } \\
\text { records review }\end{array}$ & China & RT-PCR & CT scan & $12-38$ WOG \\
\hline 20 & $\begin{array}{l}\text { Govind a et al } \\
(2020)\end{array}$ & 9 & $\begin{array}{l}\text { Retrospective Medical } \\
\text { records review }\end{array}$ & London & RT-PCR & Chest X Ray (2) & mean $39 \mathrm{WOG}$ \\
\hline 21 & Chen $\mathrm{H}$ et al (2020) & 9 & $\begin{array}{l}\text { Retrospective Medical } \\
\text { records review }\end{array}$ & China & RT-PCR & CT scan; & 33-36 weeks \\
\hline 22 & $\begin{array}{l}\text { Ferrazi E et al. } \\
(2020)\end{array}$ & 42 & $\begin{array}{l}\text { Retrospective Medical } \\
\text { records review }\end{array}$ & $\begin{array}{c}\text { Northern } \\
\text { Italy }\end{array}$ & RT-PCR & Not done & $\begin{array}{c}10=36+\text { WOG } ; \text { Intranatal }=27 \\
\text { cases; } \text { Postpartum }=5 \text { cases }\end{array}$ \\
\hline 23 & $\begin{array}{l}\text { Kayem G et al } \\
(2020)\end{array}$ & 617 & $\begin{array}{l}\text { Retrospective Medical } \\
\text { records review }\end{array}$ & France & RT-PCR & CT scan (51); & $\begin{array}{c}14-21 \mathrm{wk} 105 ; 22-31 \\
\mathrm{wk}=238 ; 32-36 \mathrm{wk}=142 ; \text { more }\end{array}$ \\
\hline
\end{tabular}

Data related to the average gestational age at diagnosis and mode of confirmation of COVID-19 is presented in Tablelof 17 pregnant women reported in the case reports, $31.25 \%$ (Kolkova et al., 2020; Tutiya et al., 2020; Romero et al., 2020) were in the gestation period of 28-32 weeks, and 43.75\% (Liu et al., 2020; Khan et al., 2020; Walczak et al., 2020) were in 37-40 weeks of gestation at the time of being diagnosed with COVID-19. All 17 (100\%) (Kolkova et al., 2020; Tutiya et al., 2020; Romero et al., 2020. Liu et al., 2020. Khan et al., 2020; Walczak et al., 2020; Koumoutsea et al., 2020. Yu et al., 2020, Alzamora et al., 2020; Hansen, Hine \& Strout, 2020) of the pregnant women were diagnosed with laboratory RT-PCR tests at the time of reporting symptoms. Chest X-ray was performed as a confirmatory assessment to rule out further lung-field involvement in 25\% (Tutiya et al., 2020; Romero et al., 2020; Koumoutsea et al., 2020) of the samples, whereas respiratory CT scans confirmed the clinical diagnosis in four out of 16 (25\%) (Kolkova et al., 2020; Tutiya, et al., 2020; Yu et al., 2020) pregnant women. No other test than RT-PCR was performed for the remaining patients and they were treated according to initial laboratory findings.

The remaining data, related to 11 retrospective clinical data reviews and one prospective review of medical records discussing 795 patients, are described in cohorts. Average gestational age of samples reviewed and the mode of clinical confirmation of COVID-19 is presented in Table 2 .

Out of 795 COVID-positive pregnant women in 11 reviews, 30.4\% (Kayem et al., 2020) presented at 22 or more weeks of gestational age at the time of hospital admission. Only 4.2\% (Zeng et al., 2020, Antoun et al., 2020; Yu et al., 2020; Nie et al., 2020; Hantoushzadeh et 
al., 2020., Liu et al., (2020); Govind et al., 2020. Ferrazzi et al., 2020; Kayem et al., C., 2020) of women reported at term, whereas $3.4 \%$ were diagnosed as COVID-positive during the intranatal period and $0.6 \%$ (Ferrazzi et al., 2020) were identified during a hospital stay in the postnatal period. All patients (Zeng et al., 2020; Antoun et al., 2020; Yu et al., 2020., Nie et al., 2020; Hantoushzadeh et al., 2020., Liu, et al., 2020, Govind et al., 2020. Ferrazzi, et al., 2020; Kayem et al., 2020. Xuet al., 2020).

Cao et al., (2020) confirmed SARS COVID positive using RT-PCR laboratory confirmation. For 108 (Zeng, et al., 2020, Yu et al., 2020., Liu et al., 2020, Kayem et al., 2020, Cao et al., 2020; Chen et al., 2020) patients, confirmation of diagnosis and respiratory involvement was confirmed with a chest CT scan after hospital admission. Chest X-ray was performed for 30 (Govind, 2020; Xu et al., 2020) patients to clinically diagnose COVID-19 after hospital admission. For remaining patients, no clinical confirmation of diagnosis was performed and they were treated only on the basis of laboratory diagnosis of COVID-19.

Table 2: Pregnancy Outcome during COVID $19 n=812$

\begin{tabular}{|c|c|c|c|c|c|c|c|c|}
\hline Sl No & $\begin{array}{l}\text { Study } \\
\text { ID }\end{array}$ & (n) & Country & $\begin{array}{l}\text { Research } \\
\text { Method }\end{array}$ & $\begin{array}{l}\text { Mode of } \\
\text { Delivery }\end{array}$ & $\begin{array}{c}\text { Reason for } \\
\text { Cs }\end{array}$ & Maternal Outcome & Fetal Outcome \\
\hline 1 & $\begin{array}{l}\text { Koumout } \\
\text { sea EV et } \\
\text { al }(2020)\end{array}$ & 2 & China & $\begin{array}{l}\text { case study } \\
\text { reviews }\end{array}$ & $\mathrm{CS}$ & \begin{tabular}{|c|} 
1st patient: \\
Multidisciplin \\
ary team \\
decision to \\
manage \\
COVID 19 \\
and prevent \\
vertical \\
transmission. \\
2nd patient: \\
Non \\
reassuring \\
fetal heart \\
rate.
\end{tabular} & 1st patient: $\mathrm{PPH}$ & $\begin{array}{c}1 \mathrm{st} \\
\text { baby=Healthy } \\
\text { male infant } \\
\text { Apgar } 9 / 9 \\
\text { 2nd baby: Non } \\
\text { reassuring fetal } \\
\text { heart rate } \\
\text { Apgar: } 4,2,7 \text { at } \\
1,5 \text { and } 10 \text { min }\end{array}$ \\
\hline 2 & $\begin{array}{c}\text { Alzamor } \\
\text { a MC et } \\
\text { al (2020) }\end{array}$ & 1 & China & $\begin{array}{c}\text { case study } \\
\text { reviews }\end{array}$ & $\mathrm{CS}$ & $\begin{array}{l}\text { Respiratory } \\
\text { failure }\end{array}$ & $\begin{array}{c}\text { Respiratory failure } \\
\text { leading to } \\
\text { mechanical } \\
\text { ventilation and } \\
\text { preterm delivery }\end{array}$ & $\begin{array}{c}\text { APGAR } 6 \text { and } 8 \\
\text { at } 1 \text { and } 5 \text { min } \\
\text { Reported } \\
\text { COVID } 19 \\
\text { Positive } 16 \text { and } \\
48 \text { hours after } \\
\text { birth }\end{array}$ \\
\hline 3 & $\begin{array}{l}\text { Liu W } \\
(2020)\end{array}$ & 3 & China & $\begin{array}{l}\text { case study } \\
\text { reviews }\end{array}$ & $2 \mathrm{CS} ; 1 \mathrm{SVD}$ & $\begin{array}{c}\text { Not } \\
\text { mentioned }\end{array}$ & $\begin{array}{l}\text { Normal Intranatal } \\
\text { and postnatal events }\end{array}$ & $\begin{array}{l}\text { Normal. No } \\
\text { abnormalities } \\
\text { reported. }\end{array}$ \\
\hline 4 & $\begin{array}{l}\text { Khan. S } \\
\text { et al } \\
(2020)\end{array}$ & 3 & China & $\begin{array}{l}\text { case study } \\
\text { reviews }\end{array}$ & $3 \mathrm{SVD}$ & & $\begin{array}{l}\text { Normal Intranatal } \\
\text { and postnatal events }\end{array}$ & $\begin{array}{c}\text { Preterm } \\
\text { APGAR 8/9 } \\
\text { APGAR 9/10 } \\
\end{array}$ \\
\hline 5 & $\begin{array}{c}\text { Zambran } \\
\text { o et al } \\
(2020)\end{array}$ & 1 & $\begin{array}{l}\text { Central } \\
\text { America }\end{array}$ & $\begin{array}{l}\text { case study } \\
\text { reviews }\end{array}$ & SVD & & Preterm & $\begin{array}{l}\text { Normal; LBW; } \\
\text { NICU admission } \\
\text { for preterm care }\end{array}$ \\
\hline
\end{tabular}




\begin{tabular}{|c|c|c|c|c|c|c|c|c|}
\hline 6 & $\begin{array}{l}\text { Kolkova } \\
\text { Z et al } \\
(2020)\end{array}$ & 1 & Sweden & $\begin{array}{l}\text { case study } \\
\text { reviews }\end{array}$ & $\mathrm{CS}$ & $\begin{array}{l}\text { Respiratory } \\
\text { distress and } \\
\text { mechanical } \\
\text { ventilation }\end{array}$ & $\begin{array}{c}\text { Acute respiratory } \\
\text { Failure, Renal } \\
\text { failure, } \\
\text { Rhabdomyolysis, } 30 \\
\text { days ICU care with } \\
\text { mechanical } \\
\text { ventilation } \\
\end{array}$ & $\begin{array}{l}\text { APGAR: } 3 / 5 / 8 \\
\text { at } 1 / 5 / 10 \text { min } \\
\text { required manual } \\
\text { ventilation } \\
\text { COVID negative }\end{array}$ \\
\hline 7 & $\begin{array}{l}\text { Celso T } \\
\text { et al } \\
(2020)\end{array}$ & 2 & Brazil & $\begin{array}{c}\text { case study } \\
\text { reviews }\end{array}$ & $\mathrm{CS}$ & $\begin{array}{l}1 \text { st patient: } \\
\text { CS due to } \\
\text { cardiovascula } \\
\text { r instability } \\
\text { 2nd patient: } \\
\text { CS due to } \\
\text { respiratory } \\
\text { failure, } \\
\text { intubation } \\
\text { and } \\
\text { mechanical } \\
\text { ventilation }\end{array}$ & $\begin{array}{c}\text { 1st patient: } \\
\text { Pulmonary } \\
\text { Microthrombi; } \\
\text { discharged stable 15 } \\
\text { days after ICU } \\
\text { care 2nd } \\
\text { patient clinically } \\
\text { stable with } 7 \text { days of } \\
\text { ICU care, discharged } \\
\text { stable after } 15 \text { days }\end{array}$ & $\begin{array}{c}\text { 1st baby: } \\
\text { APGAR } 1 / 2 \text { at } \\
\text { 1/5min Neonatal } \\
\text { death after } 9 \text { hrs } \\
\text { 2nd Baby: } \\
\text { APGAR } 7 / 9 \text { at } \\
1 / 5 \text { min NICU } \\
\text { care, COVID } \\
\text { negative. } \\
\text { Neonate } \\
\text { discharged from } \\
\text { hospital after } 67 \\
\text { days }\end{array}$ \\
\hline 8 & $\begin{array}{c}\text { Yu Y et } \\
\text { al. }(2020)\end{array}$ & 1 & China & $\begin{array}{c}\text { case study } \\
\text { reviews }\end{array}$ & SVD & & $\begin{array}{l}\text { Preterm Labour: } \\
\text { Rapidly developed } \\
\text { respiratory distress } \\
\text { after SVD }\end{array}$ & $\begin{array}{c}\text { Healthy } \\
\text { Newborn, SARS } \\
\text { negative; }\end{array}$ \\
\hline 9 & $\begin{array}{l}\text { Andrew } \\
\text { et al } \\
9(2020)\end{array}$ & 1 & Australia & $\begin{array}{c}\text { case study } \\
\text { reviews }\end{array}$ & SVD & & $\begin{array}{c}\text { Uneventful; } \\
\text { Discharged after } 10 \\
\text { days; COVID } \\
\text { negative }\end{array}$ & $\begin{array}{l}\text { APGAR 9/9; } \\
\text { COVID } \\
\text { Negative }\end{array}$ \\
\hline 10 & $\begin{array}{l}\text { Hansen } \\
\text { et al } \\
(2020)\end{array}$ & 1 & USA & $\begin{array}{c}\text { case study } \\
\text { reviews }\end{array}$ & $\mathrm{CS}$ & $\begin{array}{l}\text { Uncontrolled } \\
\text { Hypertension }\end{array}$ & $\begin{array}{c}\text { Endometriosis } \\
\text { treated with } \\
\text { antibiotics for } 7 \\
\text { days; Acute renal } \\
\text { injury; ICU on day } \\
10 \text { for worsening } \\
\text { hypoxia; Discharge } \\
\text { after } 17 \text { days after } \\
\text { COVID negative } \\
\text { results }\end{array}$ & $\begin{array}{l}\text { Normal. No data } \\
\text { presented }\end{array}$ \\
\hline 11 & $\begin{array}{c}\text { Gonzaile } \\
\text { z et al } \\
(2020)\end{array}$ & 1 & NA & $\begin{array}{c}\text { case } \\
\text { study } \\
\text { reviews }\end{array}$ & $\mathrm{CS}$ & $\begin{array}{l}\text { Respiratory } \\
\text { distress }\end{array}$ & $\begin{array}{l}\text { Preterm; Normal } \\
\text { Postnatal period; } \\
\text { Lymphopenia }\end{array}$ & $\begin{array}{l}\text { Preterm, NICU } \\
\text { care, COVID } \\
\text { negative }\end{array}$ \\
\hline 12 & $\begin{array}{l}\text { Liu D.et } \\
\text { al (2020) }\end{array}$ & 15 & China & $\begin{array}{l}\text { Retrospec } \\
\text { tive } \\
\text { medical } \\
\text { records } \\
\text { reviews }\end{array}$ & $\begin{array}{c}10 \mathrm{CS} \\
1 \mathrm{SVD} \\
4 \text { patients } \\
\text { still } \\
\text { pregnant }\end{array}$ & $\begin{array}{l}\text { Reason for } \\
\text { CS not } \\
\text { mentioned }\end{array}$ & $\begin{array}{l}12 \text { patients: } \\
\text { Lymphopenia }\end{array}$ & $\begin{array}{l}\text { No neonatal } \\
\text { abnormality } \\
\text { reported }\end{array}$ \\
\hline
\end{tabular}

46 | VOL. 12 (4) April 2021 | THE MALAYSIAN JOURNAL OF NURSING 


\begin{tabular}{|c|c|c|c|c|c|c|c|c|}
\hline 13 & $\begin{array}{l}\text { Chen } \mathrm{H} \\
\text { et al } \\
(2020)\end{array}$ & $\begin{array}{l}\text { Ch } \\
\text { ina }\end{array}$ & 9 & $\begin{array}{l}\text { Retrospec } \\
\text { tive } \\
\text { medical } \\
\text { records } \\
\text { reviews }\end{array}$ & $\mathrm{CS}$ & $\begin{array}{l}\text { COVID } 19 \text { as } \\
\text { reason in all; } \\
\text { PROM: 02; } \\
\text { Previous } \\
\text { CS:01; Pre- } \\
\text { eclampsia: } \\
01 ; \text { Fetal } \\
\text { distress } 01\end{array}$ & $\begin{array}{l}5 \text { patients had } \\
\text { lymphopenia } \\
4 \text { preterm labour at } \\
36-39 \text { weeks of } \\
\text { gestation. } \\
1 \text { pregnancy } \\
\text { complicated with } \\
\text { preeclampsia }\end{array}$ & $\begin{array}{l}2 \text { patients Fetal } \\
\text { distress } \\
2 \text { preterm } \\
\text { neonates with } \\
\text { lower birth } \\
\text { weight }\end{array}$ \\
\hline \multirow[t]{2}{*}{14} & $\begin{array}{c}\text { Ferrazi E } \\
\text { et } \\
\text { al.(2020) }\end{array}$ & 42 & $\begin{array}{l}\text { Northern } \\
\text { Italy }\end{array}$ & $\begin{array}{l}\text { Retrospec } \\
\text { tive } \\
\text { medical } \\
\text { records } \\
\text { reviews }\end{array}$ & $\begin{array}{c}24 \text { SVD } \\
\text { Elective CS } \\
18\end{array}$ & \begin{tabular}{|c|}
8 Non \\
COVID \\
reason 10: \\
Respiratory \\
Distress due \\
to COVID 19
\end{tabular} & $\begin{array}{l}\text { 1- Severe PPH } \\
2 \text { cases preterm } \\
\text { delivery } \\
\text { Admission to critical } \\
\text { care unit due to } \\
\text { respiratory distress } \\
04 \\
\text { Oxygen support } 07\end{array}$ & $\begin{array}{l}2 \text { newborns } \\
\text { tested positive } \\
\text { for COVID } 19 \\
\text { due to } \\
\text { breastfeeding } \\
\text { without mask } \\
1 \text { Newborn } \\
\text { Positive related } \\
\text { to vaginal } \\
\text { operative } \\
\text { delivery }\end{array}$ \\
\hline & & & & & & & & $\begin{array}{l}3 \text { admitted to } \\
\text { NICU for } \\
\text { respiratory } \\
\text { distress }\end{array}$ \\
\hline 15 & $\begin{array}{l}\text { Kayem } \\
\text { G et al } \\
(2020)\end{array}$ & $\begin{array}{c}61 \\
7\end{array}$ & France & $\begin{array}{l}\text { Retrospec } \\
\text { tive } \\
\text { medical } \\
\text { records } \\
\text { reviews }\end{array}$ & $\begin{array}{l}\text { Delivered }= \\
181 ; \mathrm{CS} \\
=83 / 181 ; \\
\mathrm{SVD}=98\end{array}$ & $\begin{array}{c}\mathrm{CS}=45 \text { for } \\
\text { COVID } 19 \\
\text { Other } \\
\text { obstetrical } \\
\text { reason }=38\end{array}$ & $\begin{array}{c}\text { Fetal loss at } 14-21 \\
\text { weeks }=5 / 181 \\
\text { Preterm }=50 / 181 \\
\text { IUD }=7 / 181 \text { Maternal } \\
\text { death }=018 \text { multiple } \\
\text { pregnancy with } 03 \\
\text { triplets }\end{array}$ & $\begin{array}{c}\text { COVID } 19 \\
\text { Positive } 02 / 190 \\
\text { NICU admission } \\
37 / 190 \text { Neonatal } \\
\text { death } 01 / 190\end{array}$ \\
\hline 16 & $\begin{array}{l}\text { Antoun } \\
\text { Let } \\
\text { al(2020) }\end{array}$ & 23 & UK & $\begin{array}{l}\text { Retrospec } \\
\quad \text { tive } \\
\text { medical } \\
\text { records } \\
\text { reviews }\end{array}$ & $\begin{array}{c}\mathrm{CS}=16 \\
\mathrm{SVD}=03 \\
\text { MISCARRI } \\
\text { AGE }=01 \\
\text { NOT } \\
\text { DELIVERE } \\
\text { D AT TIME } \\
\text { OF DATA } \\
\text { COLLECTI } \\
\text { ON } 03 \\
\end{array}$ & \begin{tabular}{|c|}
3 Elective \\
CS, 13 \\
Emergency \\
CS $(2$ Fetal \\
distress, 4 \\
delay and \\
failure to \\
progress in \\
labour, 7 \\
maternal \\
request/PPRO \\
$\mathrm{M} /$ sepsis
\end{tabular} & $\begin{array}{l}\text { Preterm delivery (7), } \\
\text { Reduced Fetal } \\
\text { Movement (6), Pre } \\
\text { term Premature } \\
\text { ROM (4), } \\
\text { preeclampsia (2), } \\
\text { HELLP (2), DIC (2), } \\
\text { OC (1), fetal distress } \\
\text { (1) Meconium (2) } \\
\text { Miscarriage (1) PPH } \\
\text { (1), ECMO (1), } \\
\text { death (1) }\end{array}$ & $\begin{array}{c}\text { Infected } \\
\text { Bacterial } \\
\text { pneumonia (1), } \\
\text { fetal asphyxia } \\
\text { (Resuscitated } \\
\text { using positive } \\
\text { pressure/endotra } \\
\text { cheal tube) (1) }\end{array}$ \\
\hline 17 & $\begin{array}{l}\mathrm{Nan} Y u \\
\text { et al } \\
(2020)\end{array}$ & 7 & China & $\begin{array}{l}\text { Retrospec } \\
\text { tive } \\
\text { medical } \\
\text { records } \\
\text { reviews }\end{array}$ & $\mathrm{CS}$ & COVID 19 & $\begin{array}{c}3 \text { patients had } \\
\text { Uterine scarring; NO } \\
\text { ICU admissions }\end{array}$ & $\begin{array}{l}3 \text { tested positive; } \\
\text { kept in NICU. } \\
\text { Discharged at } 2 \\
\text { weeks and } \\
\text { COVID negative }\end{array}$ \\
\hline
\end{tabular}




\begin{tabular}{|c|c|c|c|c|c|c|c|c|}
\hline 18 & $\begin{array}{c}\mathrm{Xu} \mathrm{L} \text { et } \\
\text { al }(2020)\end{array}$ & 5 & China & $\begin{array}{l}\text { Retrospec } \\
\text { tive } \\
\text { medical } \\
\text { records } \\
\text { reviews }\end{array}$ & $4 \mathrm{CS} 1 \mathrm{SVD}$ & $\begin{array}{l}4 \text { CS due to } \\
\text { Viral } \\
\text { pneumonia; }\end{array}$ & $\begin{array}{l}\text { Lymphopenia in all } \\
\text { patients Uneventful }\end{array}$ & $\begin{array}{l}\text { Normal; all } \\
\text { COVID } \\
\text { negative; }\end{array}$ \\
\hline 19 & $\begin{array}{l}\text { Cao D et } \\
\text { al (2020) }\end{array}$ & 10 & China & $\begin{array}{l}\text { Retrospec } \\
\text { tive } \\
\text { medical } \\
\text { records } \\
\text { reviews }\end{array}$ & $\begin{array}{c}2 \\
\text { Emergency } \\
\text { CS; } 6 \\
\text { Elective CS; } \\
2 \text { SVD }\end{array}$ & $\begin{array}{c}\text { 2 CS: } \\
\text { Placental } \\
\text { separation 03: } \\
\text { Preterm 03: } \\
\text { COVID 19 }\end{array}$ & $\begin{array}{l}3 \text { preterm ;4 PROM; } \\
1 \text { Placental } \\
\text { separation;2 } \\
\text { postpartum fever; 06 } \\
\text { Lymphopenia; 06 } \\
\text { Mycoplasma } \\
\text { infection }\end{array}$ & $\begin{array}{l}03 \text { Premature; all } \\
\text { COVID negative }\end{array}$ \\
\hline 20 & $\begin{array}{l}\text { Zeng Y } \\
\text { et al } \\
(2020)\end{array}$ & 16 & China & $\begin{array}{l}\text { Retrospec } \\
\text { tive } \\
\text { medical } \\
\text { records } \\
\text { reviews }\end{array}$ & $\begin{array}{c}\text { CS12; SVD: } \\
04 ;\end{array}$ & $\begin{array}{l}\text { CS for non } \\
\text { COVID } \\
\text { reason; } 3 \text { CS } \\
\text { for Preterm; } \\
\text { 01 CS for } \\
\text { Cardiac } \\
\text { disorder in } \\
\text { Pregnancy }\end{array}$ & $\begin{array}{l}\text { PROM 03; Preterm } \\
\text { 03; Polyhydramnios } \\
\text { 01; IUGR } 01\end{array}$ & $\begin{array}{c}\text { Fetal } \\
\text { Macrosomia 01; } \\
\text { All COVID } \\
\text { negative }\end{array}$ \\
\hline 21 & $\begin{array}{l}\text { Nie R, et } \\
\text { al }(2020)\end{array}$ & 33 & China & $\begin{array}{l}\text { Retrospec } \\
\text { tive } \\
\text { medical } \\
\text { records } \\
\text { reviews }\end{array}$ & $\begin{array}{l}22 \text { CS 5 } \\
\text { SVD 5 } \\
\text { Pregnancy } \\
\text { ongoing at } \\
\text { the time of } \\
\text { manuscript } \\
\text { writing 01 } \\
\text { Induced } \\
\text { Abortion }\end{array}$ & $\begin{array}{c}\text { Hypertension } \\
02 ; \\
\text { GDM }=02 ; \\
\text { Preterm } 01 ; \\
\text { Fetal distress } \\
: 04\end{array}$ & $\begin{array}{l}\text { 10: preterm labour } \\
\text { and 3: PROM }\end{array}$ & $\begin{array}{l}10 \text { Preterm, } 1 \\
\text { tested positive } \\
\text { for } \\
\text { SARSCOVID- } \\
\text { 19 4: fetal } \\
\text { distress }\end{array}$ \\
\hline 22 & $\begin{array}{c}\text { Hantoush } \\
\text { Zadeh } \mathrm{S}, \\
\text { et al } \\
(2020)\end{array}$ & 9 & Iran & $\begin{array}{l}\text { Retrospec } \\
\text { tive } \\
\text { medical } \\
\text { records } \\
\text { reviews }\end{array}$ & \begin{tabular}{|c|} 
06CS 1SVD \\
02 Not \\
delivered
\end{tabular} & $\begin{array}{l}05 \mathrm{CS} \text { due to } \\
\text { ARDS related } \\
\text { to COVID; } \\
01 \mathrm{CS} \text { due to } \\
\text { twins }\end{array}$ & $\begin{array}{l}07 \text { Maternal death; } \\
02 \text { ARDS and } \\
\text { mechanical } \\
\text { ventilation; } \\
\text { improved after } \\
\text { treatment }\end{array}$ & $\begin{array}{c}\text { Fetal Death }=05 \\
02: \text { Neonatal } \\
\text { death All } \\
\text { COVID } \\
\text { Negative }\end{array}$ \\
\hline 23 & $\begin{array}{c}\text { Govind a } \\
\text { et } \\
\text { al }(2020)\end{array}$ & 9 & London & $\begin{array}{l}\text { Retrospec } \\
\quad \text { tive } \\
\text { medical } \\
\text { records } \\
\text { reviews }\end{array}$ & $\begin{array}{l}1 \mathrm{SVD} ; 08 \\
\mathrm{CS}\end{array}$ & $\begin{array}{c}2 \text { Emergency } \\
\text { CS } \\
\text { Respiratory } \\
\text { distress; 1 } \\
\text { Emergency } \\
\text { CS for Non- } \\
\text { reassuring } \\
\text { CTG; } 01 \\
\text { Elective CS } \\
\text { for Breech; } \\
\text { 04 Elective } \\
\text { CS for } \\
\text { previous CS } \\
\text { and maternal } \\
\text { request }\end{array}$ & $\begin{array}{c}\text { patient } 1= \\
\text { Lymphopenia, } \\
\text { ECMO; Patient } 2= \\
\text { Mechanical } \\
\text { ventilation required } \\
\text { for } 4 \text { days postnatal; } \\
\text { others stable and } \\
\text { managed well }\end{array}$ & $\begin{array}{c}1=\text { COVID } \\
\text { positive } 1=\text { ICU } \\
\text { admission }\end{array}$ \\
\hline
\end{tabular}


The pregnancy outcomes identified from the reviews is analyzed as maternal and foetal outcomes separately and is given below. An overall summary of outcomes in mother and fetus among pregnant women who tested COVID-19 positive is presented in the case reports in table 2 .

Among 17 patients, 59\% (Kolkova, et al., 2020., Tutiya et al., 2020; Romero et al., 2020, Koumoutsea et al., 2020, Yu et al., 2020, Alzamora et al., 2020) had CS as the mode of delivery, and 41\% (Liu et al., 2020; Walczak, et al., 2020.,Yu et al., 2020; Zambrano et al., 2020) underwent spontaneous vaginal delivery (SVD). Five out of 10 patients underwent CS due to respiratory failure related to their COVID-19-positive status; (Kolkova, et al., 2020., Tutiya et al., 2020., Koumoutsea et al., 2020; Alzamora et al., 2020) three out of ten patients underwent CS for obstetrical/non-COVID reasons, while no data was presented for the remaining two patients (Liu et al., 2020).

In terms of maternal outcomes; five of the 17 (Romero et al., 2020., Alzamora, et al., 2020; Zambrano et al., 2020) patients had a preterm delivery, four (Liu et al., 2020. Khan et al., 2020.,Walczak et al., 2020) had a normal, uneventful labour process; two (Koumoutsea et al., 2020., Hansen, Hine, \& Strout, 2020) patients reported $\mathrm{PPH}$, two more reported endometriosis; while postnatal rhabdomyolysis, lymphopenia and respiratory microthrombi were each reported by one (Kolkova et al., 2020., Romero et al., 2020) patient. All patients were treated and discharged healthy, after confirmation of COVID-19-negative status.

Neonatal outcomes are explained in terms of onemin and five-min Apgar scores, as well as COVID-19 status after birth. Thirty percent (Kolkova et al., 2020; Tutiya et al., 2020; Koumoutsea et al., 2020) and 10\% (Kolkova et al., 2020., Tutiya et al., 2020) of neonates presented with an Apgar score below five at one min and five min respectively, while an Apgar score above seven was observed in $60 \%$ (Tutiya et al., 2020., Liu et al., 2020, Walczak et al., 2020; Koumoutsea, et al., 2020) of neonates at one min and 80\% (Kolkova et al., 2020; Tutiya et al 2020; Khan et al., 2020; Walczak et al., 2020. Koumoutsea et al., 2020; Alzamora et al., 2020) of neonates at five mins. Apgar details were not presented for three infants, whereas one (Tutiya et al., 2020) neonatal deaths were reported, nine hours after birth, which was related to preterm status and respiratory failure. Out of 13 new-borns, only one baby born by CS was reported COVID-19 positive, 16 and 48 hours after birth (Alzamora et al., 2020).

Among the 795 reviewed reports, 25.78\% (Zeng et al., 2020; Antoun et al., 2020; Yu et al., 2020., Nie et al., 2020; Hantoushzadeh et al., 2020., Liu et al., 2020; Govind et al., 2020; Ferrazzi et al., 2020; Kayem et al., 2020. Xu et al., 2020; Caob et al., 2020., Xu et al., 2020; Cao et al., 2020) of patients underwent CS; 56.6\% (Antoun et al., 2020; Nie et al., 2020; Hantoushzadeh et al., 2020; Liu et al., 2020) were still pregnant at the time of review and 17.6\% (Zeng et al., 2020, Antoun et al., 2020., Nie et al., 2020; Hantoushzadeh et al., 2020; Liu et al., 2020; Govind et al., 2020; Ferrazzi et al., 2020, Kayem et al., 2020; Xu et al., 2020; Cao et al., 2020) had SVD. Forty-six per cent (90 of 194 patients) (Zeng et al., 2020; Yu et al., 2020; Hantoushzadeh et al., 2020; Govind et al., 2020; Ferrazzi et al., 2020; Kayem et al., 2020; Cao et al., 2020) underwent CS for reasons connected to COVID-19, whereas $48.5 \%$ (94 of the 194) (Zeng et al., 2020; Nie et al., 2020. Hantoushzadeh et al., 2020; Liu et al.,2020; Ferrazzi et al., 2020; Kayem et al., 2020; Xu, et al., 2020; Cao et al., 2020) had CS for nonCOVID, obstetrical reasons. For 10 patients, the reason for CS was not presented in the report (Liu et al., 2020; Govind, et al., 2020).

Twenty-nine patients reported lymphopenia in the postnatal period (Liu, et al., 2020; Govind, et al., 2020; $\mathrm{Xu}$, et al., 2020; Cao, et al., 2020; Chen et al., 2020) 76 (Zeng, et al., 2020; Antoun, et al., 2020; Nie, et al., 2020; Ferrazzi, et al., 2020; Kayem et al., 2020; Cao et al., 2020) had preterm labour, two (Antoun, et al., 2020; Ferrazzi et al., 2020) reported severe PPH, eight (Antoun et al., 2020; Hantoushzadeh et al., 2020; Kayem, et al., 2020) suffered maternal death related to acute respiratory distress syndrome and postnatal complications, and three patients had uterine scarring in the postnatal period ( $\mathrm{Yu}$, et al., 2020).

A total of 14 new-born tested COVID-19 positive (Yu, et al., 2020; Ferrazzi et al., 2020; Kayem, et al., 2020). Eight patient cases reported foetal/neonatal death (Hantoushzadeh et al., 2020; Kayem, et al., 2020) and 42 neonates were admitted to NICU with clinical manifestations of foetal distress (Antoun et al., 2020; Nie, et al., 2020; Ferrazzi, et al., 2020; Chen et al., 2020)

\section{DISCUSSION}

This systematic review showed a moderate effect of COVID-19 in pregnancy on outcomes in mother and 
newborn. COVID-19 is a highly infectious disease with maximum incidence being reported among people with weak immune status as well as multiple comorbidities (Lipsitch, et al., 2020; Zhou et al., 2020; Jordan, Adab \& Cheng, 2020). As the COVID-19 pandemic continues to have worldwide effects, the most affected group seems to involve a majority of pregnant women.

Cases of COVID-19 infection in pregnant women have been described in multiple reports and reviews from around the world. Obstetricians and health care workers globally have raised concerns over expected risk of severe disease, viral transmission through vertical route and complications in mother, fetus and newborn (Dong et al., 2020). Primary aim of this systematic review was to describe the current scientific evidence relating to COVID-19 in pregnancy and newborn period.

Lack of mandatory testing for SARS-CoV-2 during pregnancy (understanding that most of pregnant women infected with SARS-CoV-2 were tested only after reporting symptoms while in hospital), and minimal availability of standardized antenatal surveillance, management, timing and type of delivery among women with COVID-19 was observed in all the reports reviewed. The recent updates and modifications in maternity care services worldwide may have an impact upon COVID-19's effect on outcomes in maternal and perinatal period during pregnancy, which are unmeasured in this review. A significant number of pregnant women presented in the third trimester with COVID-19 symptoms and then delivered, so we were unable to measure meaningfully the maternal and fetal outcome in terms of the association between COVID-19 infection and different gestational ages.

As shown by the studies included in this review, among 362 neonates, $22.37 \%$ of were preterm newborn and $1.3 \%$ of patients reported pregnancy loss at different initial trimesters. Of the 362 women who had delivered by the time of review completion, 215 had a CS delivery and 147 had a vaginal delivery. According to some previous reviews of similar data regarding the maternal outcome, there is some similarity between the proportion of C-section deliveries in patients with COVID-19 was $45 \%$ almost closer to that reported here of $59.3 \%$, with both presenting an average proportion of $\mathrm{C}$-section deliveries. However, a $23 \%$ rate of preterm delivery was described in a parturient with COVID-19, a similar frequency as presented $22.37 \%$ and 1.35 pregnancy loss of patients with COVID-19 included in this review
(Capobianco et al., 2020).

Case reports from China (Liu, et al., 2020; Khan et al., 2020), Australia (Walczak et al., 2020) reported no adverse outcomes concerning COVID-19 and pregnancy, which was similar to report presented where few case reports reviewed from around the world showed no adverse pregnancy outcomes (Dubey et al., 2020). Our review report found 4.14\% COVID-19 infection rates among newborn, based on review of case study as well as in review of medical records done retrospectively. One neonate was reported with COVID-19 in the case studies review and 14 more were reported in the retrospective review of medical records. The majority of mothers of these neonates were shifted to an intensive care unit for respiratory distress in the postnatal period. Although it is insufficient to validate vertical transmission of infection, it is concomitant with the review findings presented by Dubey et al, in which $1 \%$ of neonates were found to be COVID-19 positive. However, more research is required to establish the relationship between COVID-19 and vertical transmission.

\section{CONCLUSION}

The coronavirus pandemic has had a major impact on health worldwide. Knowledge and associated facts about this illness have been discussed and presented faster than the rate of globalization in the past decade. Although there is a lack of substantial evidence of the infection affecting maternal and neonatal outcomes, studies prove that pregnant women and newborn children are at high risk of developing adverse outcomes. COVID-19 is highly contagious and must be considered when planning antenatal, Intranatal, postnatal, and newborn care. Pregnancy does not increase the risk of infection and pregnant women should follow the same recommendations as the rest of the general population to limit the transmission of COVID-19. However, pregnancy might increase the severity of respiratory distress in cases involving severe infection and may necessitate preterm labor and CS. The appropriate screening of pregnant mothers for COVID-19 at different trimesters is therefore essential, especially when there are comorbid medical illnesses and the presence of flu-like symptoms. This can reduce the maternal and fetal complications for a pregnant woman who is COVID-19 positive. As the disease is, continually evolving, healthcare providers should update themselves with recent developments in the vertical transmission of COVID-19 and other developments in the disease 
process to ensure that the best maternal and fetal health care is available to pregnant women who are COVID-19 positive.

\section{Conflict of Interests}

The authors declare that they have no conflict of interest.

\section{ACKNOWLEDGEMENT}

Authors would like to acknowledge the institutional administration for providing us the access to wide database, enabling us to have a review and achieve desired objective.

\section{REFERENCES}

Alzamora, M.C., Paredes, T., Caceres, D., Webb, C.M., Valdez, L.M. and La Rosa, M. (2020). Severe COVID-19 during pregnancy and possible vertical transmission. American Journal of Perinatology, 37(8), p.861.

Antoun, L., El Taweel, N., Ahmed, I., Patni, S. \& Honest, H., 2020. Maternal COVID-19 infection, clinical characteristics, pregnancy, and neonatal outcome: A prospective cohort study. European Journal of Obstetrics \& Gynecology and Reproductive Biology, 252, pp. 559-562.

Bouaziz, J., Even, M., Isnard-Bogillot, F., Vesale, E., Nikpayam, M., Mihalache, A., Krief, D., Frydman, R. \& Ayoubi, J.M. (2020). COVID-19 in pregnancy: What do we really know?. F1000Research, 9(362), p.362.

Cao, D., Yin, H., Chen, J., Tang, F., Peng, M., Li, R., Xie, H., Wei, X., Zhao, Y. \& Sun, G. (2020). Clinical analysis of ten pregnant women with COVID-19 in Wuhan, China: A retrospective study. International Journal of Infectious Diseases. 95: pp 294-300.

Capobianco, G., Saderi, L., Aliberti, S., Mondoni, M., Piana, A., Dessole, F., Dessole, M., Cherchi, P.L., Dessole, S. \& Sotgiu, G. (2020). COVID-19 in pregnant women: A systematic review and meta-analysis. European Journal of Obstetrics \& Gynecology and Reproductive Biology, 252, pp 543-558.

Cevallos, M., Egger, M. \& Moher, D. (2014). STROBE (STrengthening the Reporting of OBservational studies in Epidemiology). Guidelines for Reporting Health Research: a user's Manual, pp.169-79.

Chen, H., Guo, J., Wang, C., Luo, F., Yu, X., Zhang, W., Li, J., Zhao, D., Xu, D., Gong, Q. \& Liao, J. (2020). Clinical characteristics and intrauterine vertical transmission potential of COVID-19 infection in nine pregnant women: a retrospective review of medical records. The Lancet, 395(10226), pp 809-815.

Dashraath, P., Jeslyn, W.J.L., Karen, L.M.X., Min, L.L., Sarah, L., Biswas, A., Choolani, M.A., Mattar, C. \& Lin, S.L. (2020). Coronavirus disease 2019 (COVID-19) pandemic and pregnancy. American Journal of Obstetrics and Gynecology. 222 (6) pp 521-531.

Dong, L., Tian, J., He, S., Zhu, C., Wang, J., Liu, C. \& Yang, J., 2020. Possible vertical transmission of SARS-CoV-2 from an infected mother to her newborn. Jama, 323 (18), pp 1846-1848.

Dubey, P., Reddy, S., Manuel, S. \& Dwivedi, A.K. (2020). Maternal and neonatal characteristics and outcomes among COVID-19 infected women: An updated systematic review and meta-analysis. European Journal of Obstetrics \& Gynecology and Reproductive Biology, 252, pp 490-501.

Ferrazzi, E., Frigerio, L., Savasi, V., Vergani, P., Prefumo, F., Barresi, S., Bianchi, S., Ciriello, E., Facchinetti, F., Gervasi, M.T. \& Iurlaro, E. (2020). Vaginal delivery in SARS-CoV-2-infected pregnant women in Northern Italy: a retrospective analysis. BJOG: An International Journal of Obstetrics \& Gynaecology, 127: 1116-1121.

Gagnier, J.J., Kienle, G., Altman, D.G., Moher, D., Sox, H., Riley, D. \& CARE Group (2013). The CARE guidelines: consensus-based clinical case reporting guideline development. Journal of Medical Case Reports, 7(1), p.223.

Govind, A., Essien, S., Karthikeyan, A., Fakokunde, A., Janga, D., Yoong, W. \& Nakhosteen, A., 2020. Re: Novel 
Coronavirus COVID-19 in late pregnancy: Outcomes of first nine cases in an inner-city London hospital. European Journal of Obstetrics, Gynecology, and Reproductive Biology.251, pp 272-274.

Hansen, J.N., Hine, J. \& Strout, T.D. (2020). COVID-19 and preeclampsia with severe features at 34-weeks gestation. The American Journal of Emergency Medicine, 39, pp 252-e3.

Hantoushzadeh, S., Shamshirsaz, A.A., Aleyasin, A., Seferovic, M.D., Aski, S.K., Arian, S.E., Pooransari, P., Ghotbizadeh, F., Aalipour, S., Soleimani, Z. \& Naemi, M. (2020). Maternal death due to COVID-19. American Journal of Obstetrics and Gynecology, 223(1), pp109-e1.

Jordan, R.E., Adab, P. \& Cheng, K.K. (2020). Covid-19: risk factors for severe disease and death. BMJ, 368:m1198

Kayem, G., Lecarpentier, E., Deruelle, P., Bretelle, F., Azria, E., Blanc, J., Bohec, C., Bornes, M., Ceccaldi, P.F., Chalet, Y. \& Chauleur, C. (2020). A snapshot of the Covid-19 pandemic among pregnant women in France. Journal of Gynecology Obstetrics and Human Reproduction, 49(7), p.101826.

Khan, S., Peng, L., Siddique, R., Nabi, G., Xue, M., Liu, J. \& Han, G. (2020). Impact of COVID-19 infection on pregnancy outcomes and the risk of maternal-to-neonatal intrapartum transmission of COVID-19 during natural birth. Infection Control \& Hospital Epidemiology, 41(6), pp.748-750.

Kolkova, Z., Bjurström, M.F., Länsberg, J.K., Svedas, E., Hamer, M.A., Hansson, S.R., Herbst, A. and Zaigham, M., 2020. Obstetric and intensive-care strategies in a high-risk pregnancy with critical respiratory failure due to COVID-19: A case report. Case reports in Women's Health, 27, p.e00240.

Koumoutsea, E.V., Vivanti, A.J., Shehata, N., Benachi, A., Le Gouez, A., Desconclois, C., Whittle, W., Snelgrove, J. \& Malinowski, A.K. (2020). COVID-19 and acute coagulopathy in pregnancy. Journal of Thrombosis and Haemostasis, 18, pp 1648-1652.

Lipsitch, M., Swerdlow, D.L. \& Finelli, L. (2020). Defining the epidemiology of Covid-19—studies needed. New England Journal of Medicine, 382(13), pp 1194-1196.

Liu, D., Li, L., Wu, X., Zheng, D., Wang, J., Yang, L. \& Zheng, C. (2020). Pregnancy and perinatal outcomes of women with coronavirus disease (COVID-19) pneumonia: a preliminary analysis. American Journal of Roentgenology, 215(1), pp 1-6.

Liu, W., Wang, Q., Zhang, Q., Chen, L., Chen, J., Zhang, B., Lu, Y., Wang, S., Xia, L., Huang, L. \& Wang, K. (2020). Coronavirus disease 2019 (COVID-19) during pregnancy: a case series. Preprints 2020, 2020020373.

Moher D, Liberati A, Tetzlaff J. \& DG, The PRISMA Group (2009). Preferred Reporting Items for Systematic Reviews and Meta-Analyses: The PRISMA Statement. PLoS Med, 6(7).

Nie, R., Wang, S.S., Yang, Q., Fan, C.F., Liu, Y.L., He, W.C., Jiang, M., Liu, C.C., Zeng, W.J., Wu, J.L. \& Oktay, K. (2020). Clinical features and the maternal and neonatal outcomes of pregnant women with coronavirus disease 2019. MedRxiv.

Panahi, L., Amiri, M. \& Pouy, S. (2020). Risks of novel coronavirus disease (COVID-19) in pregnancy; a narrative review. Archives of Academic Emergency Medicine, 8(1), e34.

Qiao, J. (2020). What are the risks of COVID-19 infection in pregnant women? The Lancet, 395(10226), pp 760-762.

Rasmussen, S.A., Smulian, J.C., Lednicky, J.A., Wen, T.S. \& Jamieson, D.J. (2020). Coronavirus Disease 2019 (COVID-19) and Pregnancy: What obstetricians need to know. American Journal of Obstetrics and Gynecology, 222(5), pp 415-426

Romero, D.G., Pérez, J.O., Bautista, L.G. \& Santana-Cabrera, L. (2020). Pregnancy and perinatal outcome of a woman with COVID-19 infection. Revista Clinica Espanola, 220(8), pp 533-534. 
Schwartz, D.A. (2020). An analysis of 38 pregnant women with COVID-19, their newborn infants, and maternal-fetal transmission of SARS-CoV-2: maternal coronavirus infections and pregnancy outcomes. Archives of Pathology \& Laboratory Medicine, 144(7), pp 799-805.

Tutiya, C.T., Siaulys, M.M., Kondo, M.M., Miglioli-Galvão, L., Galvão, E.C., Pinheiro, C.C., Torloni, M.R. \& de Mello, F.B. (2020). Possible formation of pulmonary microthrombi in the early puerperium of pregnant women critically ill with COVID-19: Two case reports. Case Reports in Women's Health, 27, p e00237.

Walczak, A., Wilks, K., Shakhovskoy, R., Baird, T., Schlebusch, S., Taylor, C., Reid, D. \& Choong, K. (2020). COVID-19 in a complex obstetric patient with cystic fibrosis. Infection, Disease \& Health, 25(4), pp 239-241.

Xu, L., Yang, Q., Shi, H., Lei, S., Liu, X., Zhu, Y., Wu, Q., Ding, X., Tian, Y., Hu, Q. \& Chen, F. (2020). Clinical presentations and outcomes of SARS-CoV-2 infected pneumonia in pregnant women and health status of their neonates. Science Bulletin, 65(18), pp 1537-1542.

Yu, N., Li, W., Kang, Q., Xiong, Z., Wang, S., Lin, X., Liu, Y., Xiao, J., Liu, H., Deng, D. \& Chen, S. (2020). Clinical features and obstetric and neonatal outcomes of pregnant patients with COVID-19 in Wuhan, China: a retrospective, single-centre, descriptive study. The Lancet Infectious Diseases, 20(5), pp 559-564.

Yu, Y., Fan, C., Bian, J. \& Shen, Y. (2020). Severe COVID-19 in a pregnant patient admitted to hospital in Wuhan. International Journal of Gynecology \& Obstetrics, 150, pp 262-263.

Zaigham, M. \& Andersson, O. (2020). Maternal and perinatal outcomes with COVID-19: A systematic review of 108 pregnancies. Acta Obstetricia Et Gynecologica Scandinavica, 99(7), pp 823-829.

Zambrano, L.I., Fuentes-Barahona, I.C., Bejarano-Torres, D.A., Bustillo, C., Gonzales, G., Vallecillo-Chinchilla, G., Sanchez-Martínez, F.E., Valle-Reconco, J.A., Sierra, M., Bonilla-Aldana, D.K. \& Cardona-Ospina, J.A. (2020). A pregnant woman with COVID-19 in Central America. Travel Medicine and Infectious Disease, 36, pp101639.

Zeng, Y., Lin, L., Yan, Q., Wei, W., Yang, B.X., Huang, R., He, F. \& Chen, D. (2020). Update on clinical outcomes of women with COVID-19 during pregnancy. International Journal of Gynecology \& Obstetrics, 150(2), pp 264266.

Zhou, F., Yu, T., Du, R., Fan, G., Liu, Y., Liu, Z., Xiang, J., Wang, Y., Song, B., Gu, X. \& Guan, L. (2020). Clinical course and risk factors for mortality of adult inpatients with COVID-19 in Wuhan, China: a retrospective cohort study. The lancet, 395(10229), pp 1054-1062. 